\section{Streamlining the Modern Lab}

\author{
Radhika Subramanian \\ Cornet Technology, Inc., Springfield, VA \\ rsubramanian@cornet.com
}

\section{Introduction}

Imagine what a researcher 50 years ago would think of the technology available in today's modern research labs, where the ability to photograph and videotape live cell images in high resolution has spurred numerous advances in the sciences. Additionally, technology's shift from analog to digital image acquisition has enhanced scientific collaboration and analysis abilities, perhaps in ways our predecessors could never have imagined. However, the massive quantities of data that result from these image acquisition techniques, and subsequent storage requirements, have created unforeseen challenges. While we describe life science applications, the methods described in this article are equally applicable in physical sciences laboratories.

Certainly innovation is changing the way researchers go about their daily business. To meet the challenges of the modern bio-medical and life scientist, thought leaders are persistently testing the limits of research and design. While the modern lab is an exciting setting for the dedicated microscopy professional, it can also be fraught with tedious, time consuming, and often frustrating technological roadblocks.

Perhaps the biggest advances have been in the way researchers store, transfer, retrieve, and manage massive quantities of large image files. In some cases, labs will store up to 50 terabytes (TB) of data at one time. To put this in perspective, an average CD-ROM can roughly equal 800 MBs. Therefore, a $1 \mathrm{~TB}$ storage drive has the capacity to store information from well-over 1,000 CD's. Clearly, access to a hard drive with a $1 \mathrm{~TB}$ storage capacity saves innumerable hours spent on data transfer and management.

This article analyzes the technological and administrative challenges of scientific labs and provides an overview of how new computer hardware and software are helping scientists manage, store, and transport vital microscopic imagery.

\section{Lab Management}

Managing a busy laboratory requires a high degree of organization, coupled with the right tools to do the job well. These tools include everything from high-powered microscopes to test tube brushes, as well as the right software solutions.

\section{Resource Consumption}

Ask any good lab administrator and he/she will tell you that understanding resource consumption is the key to better lab management. To achieve this understanding, managers need secure flexible access and control of their systems. However, equipment usage is often difficult to track, not always accurate, and is frequently performed manually by the user. Currently, this process requires the mind numbing, and time consuming, review of paper logs to ensure appropriate billing. Fortunately, lab management tools are available today that facilitate and streamline this process, drastically reducing the time and effort that researchers devote to logging information. For example, Cornet Technology's (www.cornet.com) Lab Track software program tracks equipment usage for administration requirements. This software is shareable and runs on a Microsoft Windows platform. Data usage parameters are recorded 24/7 and stored in an internal database for reference, as needed, by lab administrators.

Lab Track can monitor the usage of any equipment connected to a PC. When a user logs on to a computer, the software automatically starts and tracks the usage through the generation of graphs and reports. Administrators can produce reports based on weekly, monthly, and yearly equipment usage; specific lab usage rules; individual users; groups of users within an individual lab; groups of labs; and date fields.
The software also generates comprehensive billing reports for labs where equipment usage is billable. This gives lab managers the ability to invoice for lab time based on actual usage, rather than on an approximation of time. In addition, research funding can be better allocated by analyzing usage times for specific equipment.

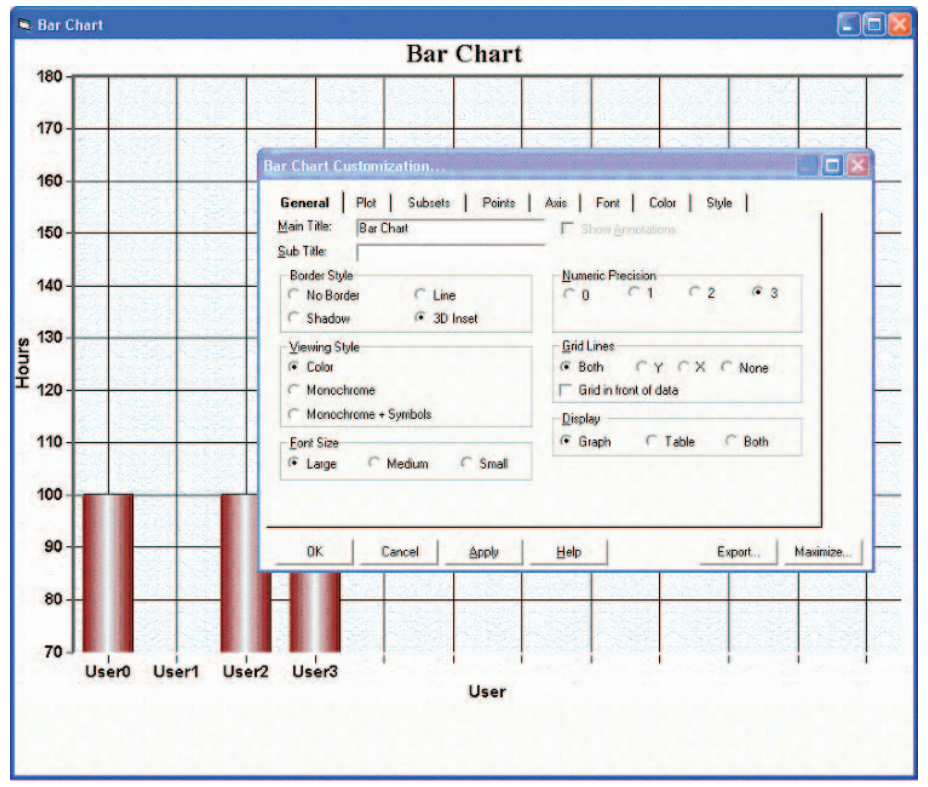

Figure 1: LabTrack monitors the usage of any laboratory equipment connected to $a$ PC and generates easy-to-read bar graphs and pie charts illustrating each user's activity. The software also allows lab managers to define rules and hours of operation by setting parameters for equipment usage, and generates comprehensive billing reports for labs where equipment usage is billable.

\section{Lab Rules}

Obstacles to equipment usage can be frustrating. In many labs, a limited amount of equipment must be utilized by many scientists, creating resource bottlenecks. Researchers often require swift access to lab tools for timely completion of their research. The facilitation of this process is an important component of the lab manager's job.

To avoid gridlock in the lab, Lab Track software allows managers to define rules and hours of operation by setting parameters for equipment usage. Once the rules are initialized, the software automatically tracks that specific information and a report is generated on demand.

\section{Data Storage \& Transfer}

When laboratory scientists capture digital still photos and video images of organisms through high-powered microscopes, such as confocal microscopes, they amass a huge amount of data quickly. Transferring this data to CD-ROMs, or other conventional storage devices, can be time consuming, often requiring several hours per day for data transfer time. Consequently, there is a critical need for a portable hardware device with the capability to store massive amounts of image data files.

\section{You Can Take It with You}

Many researchers currently utilize a one or two gigabyte flash drive to store and transfer data. Unfortunately, large image files can quickly generate data of this size, requiring scientists to spend valuable time uploading and storing information onto higher capacity storage devices. Fortunately, the large storage capabilities of the latest portable hard drives have drastically reduced the amount of time researchers spend transferring microscope image files.

\section{Grow Your Storage Capacity}

Large files, often several gigabytes in size, have the very real potential of overloading systems. In addition, maintaining and accessing extensive quantities of images, created during the research process, presents a challenge for even the most organized. 


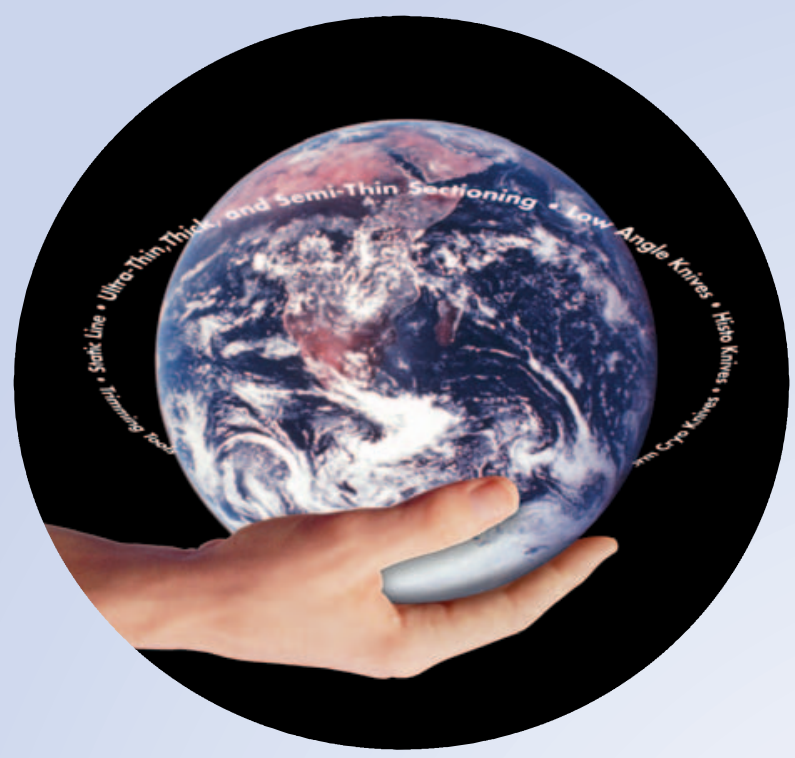

THE WORLD OF MICROSCOPY \& MICROTOMY SOLUTIONS IS WITHIN YOUR REACH WITH
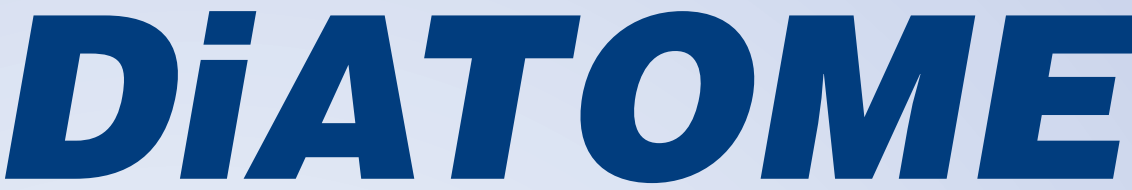

With 36 years of Developing, Innovating \& Discovering under their belts, DiATOME is the only Diamond Knife manufacturer that does not sit around waiting for technology to happen ... they make it happen leaving the other Diamond Knife manufacturers light years behind.

1970 ... DIATOME introduces the FIRST DIAMOND KNIFE.

- 1980 ... DiATOME introduces the FIRST CRYO DIAMOND KNIFE.

1983 ... DIATOME introduces the FIRST SEMI DIAMOND KNIFE FOR ALTERNATING SECTIONING.

1985 ... DiATOME introduces the FIRST LOW ANGLE (35) DIAMOND KNIFE.

1 1986 ... DIATOME introduces the FIRST "HISTO" THICK SECTIONING DIAMOND KNIFE.

1989 ... DIATOME introduces the FIRST STATIC ELIMINATOR \& CRYO DIAMOND TRIMMING TOOL. (Revolutionizing Cryo Ultramicrotomy as we know it)

1999 ... DIATOME introduces the FIRST \& ONLY CRYO-P DIAMOND KNIFE.

(A Cryo Knife with a Patented Platform for section pick up)

2001 ... DIATOME introduces the CRYO IMMUNO DIAMOND KNIFE.

(The First Cryo Knife with a Diamond Plateau: Optimized pick-up for best section quality in immuno-cytochemistry!)

\ 2004 ... DIATOME introduces the REVOLUTIONARY ULTRA SONIC DIAMOND KNIFE.

2005 ... DIATOME introduces the CRYO-TRIM $25^{\circ}$ OPTIMIZING TRIMMING WITH DIAMOND BLADES.

- 2006 ... DIATOME introduces the FIRST DIAMOND KNIVES FOR AFM AT ROOM \& LOW TEMPERATURES.

2006 ... DIATOME introduces the CRYO 25 DIAMOND KNIFE FOR SECTIONING FROZEN HYDRATED SPECIMENS.

\section{DHATOME THANKS THEIR CUSTOMERS FOR 36 YEARS OF PATRONAGE!}
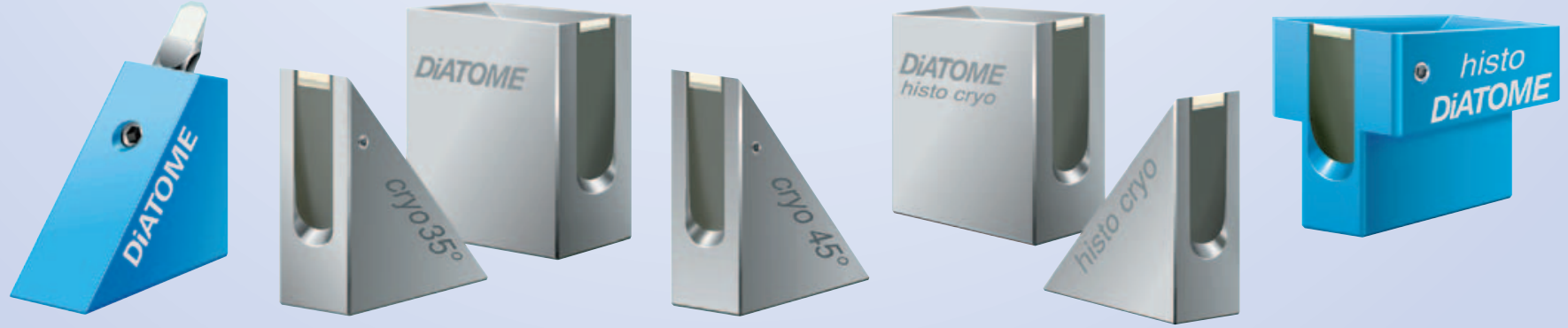

For a copy of our complete product catalog or individual brochure on any of our products, please call or write today, or visit us on the web at: www.emsdiasum.com

\section{DHATOME 1560 Industry Road P.O. Box 410 - Hatfield, PA 19440} tel:2 I5.4 I 2.8390 - fax:2 I5.4 12.8450 - email:sgkcck@aol.com -web:www.emsdiasum.com 
Currently, several major research laboratories are using the Maximus Platinum one-terabyte portable hard drive, along with the newly available Scientific Image Manager software, together or independently, to quickly store huge data files and to organize multiple microscopic image files. Researchers know that a great deal can be learned about various cells and proteins by capturing high-speed videos of them. However, some of these videos consist of up to 120 images per second. This process can quickly generate one or two gigabyte files, which can take a lot of time to upload and store. Cornet Technology's new portable one-terabyte hard drive has enabled these laboratories to significantly streamline the process of uploading, transporting, and storing large image files.

\section{Image Management Multiple File Types}

The Scientific Image Manager is a valuable tool designed to address the numerous digital im-

age file formats used at research institutions. As Windows Explorer is incapable of reading microscope image files, the Scientific Image Manager was developed to provide a single view of specialized file formats, eliminating the need to toggle between Internet Explorer and other proprietary viewer programs. The software's advanced platform-independent features increase productivity and streamline workflow; enabling scientists to use Microsoft Windows, Macintosh, or UNIX platforms.

The software allows researchers to preview thumbnail images, rather than icons, of several file types. In addition, users can add unlimited notes (e.g. information on the type of experiment they performed) for each image and folder type and view these notes along with a complete list of all image folders in one view.

This software's comprehensive user interface for managing and viewing Zeiss and other microscope image file types such as MDB, LSM, and TIFF files is a key component to streamlining work practices. In addition, Cornet Technology also has the ability to custom integrate any new image type to meet specific research criteria.

\section{Get Organized}

The Scientific Image Manager software allows researchers to view thumbnails rather then just file names. Scientific Image Manager enables researchers to organize, label, search, and view images easily, making file identification a much simpler process.

The capacity to add detailed notations and descriptions to MDB and TIFF image files provides a significant benefit to the file search and identification process. This advanced editing feature allows for more advanced file organization and comprehensive searching options. Powerful keyword and description search tools make it easy and convenient to search image files quickly and efficiently. Searches can be based on either full or truncated file names as well as keywords located in a file's notes and descriptions.

\section{Conclusion}

In the modern laboratory there are a number of technological barriers to streamlining workflow. These challenges include the storage, transfer, retrieval and management of large amounts of image files created during the research process. Currently there are a number of high capacity storage devices to address the storage management needs of the modern laboratory. These devices, combined with intelligent image management and equipment usage software, are critical

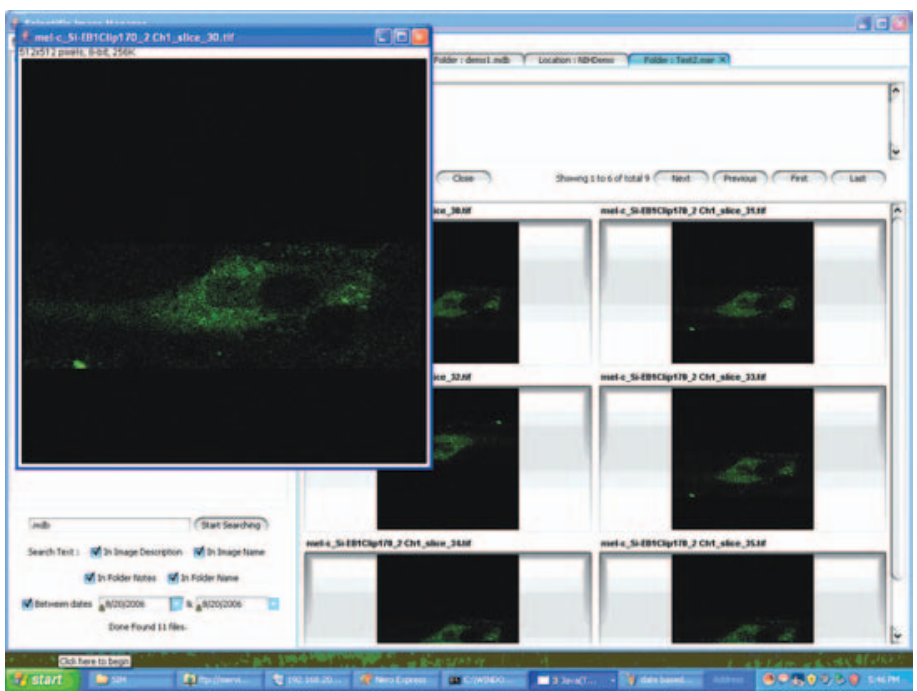

Figure 3: The Scientific Image Manager is a software solution that allows users within the biomedical industry to display and manage multiple confocal microscope image files in one format. This software has a comprehensive user interface that was developed for the management and presentation of Zeiss and all other confocal microscope image file types.

tools to streamlining the modern lab. Cornet Technology's solutions offer an integrated system that employs the Maximus Platinum oneterabyte external hard drive, the Lab Track equipment usage tracking program, and a custom designed software package called Scientific Image Manager (SIM), which displays and manages multiple microscope image files according to a lab's specific requirements.

These solutions were developed to address the unique challenges faced by today's microscopy professionals. By continuing to work closely with scientists to address their most challenging technology based issues, modern innovations will continue to change the face of laboratory research.

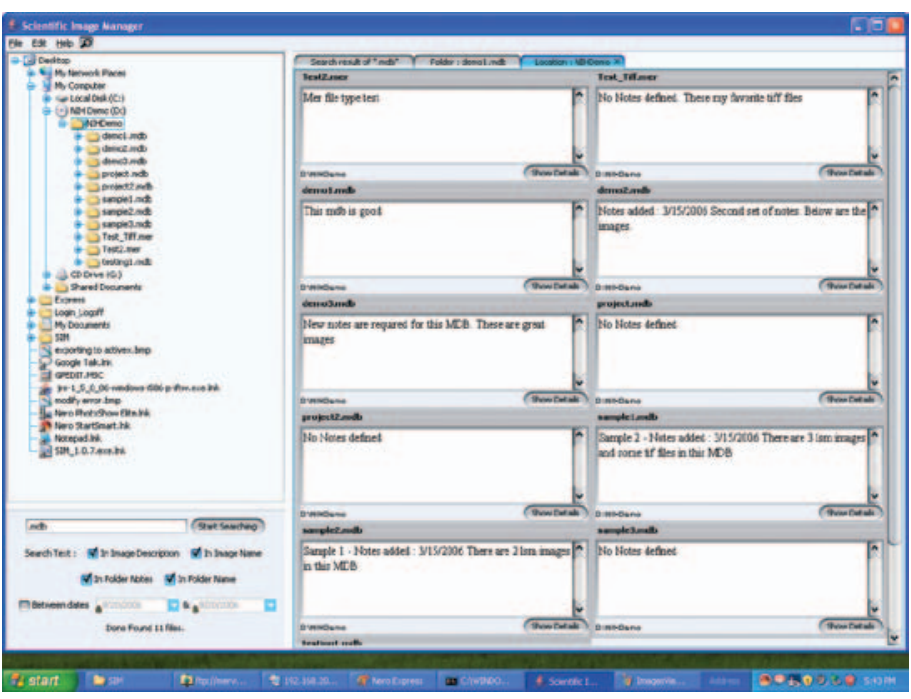

Figure 4: Scientific Image Manager allows for the addition of detailed notations and descriptions to MDB and TIFF image files. This advanced editing feature allows for more advanced file organization and comprehensive searching options. Searches can be based on either full or truncated file names as well as keywords located in a file's notes and descriptions. 


\section{Advanced}

\section{Detector}

Technology

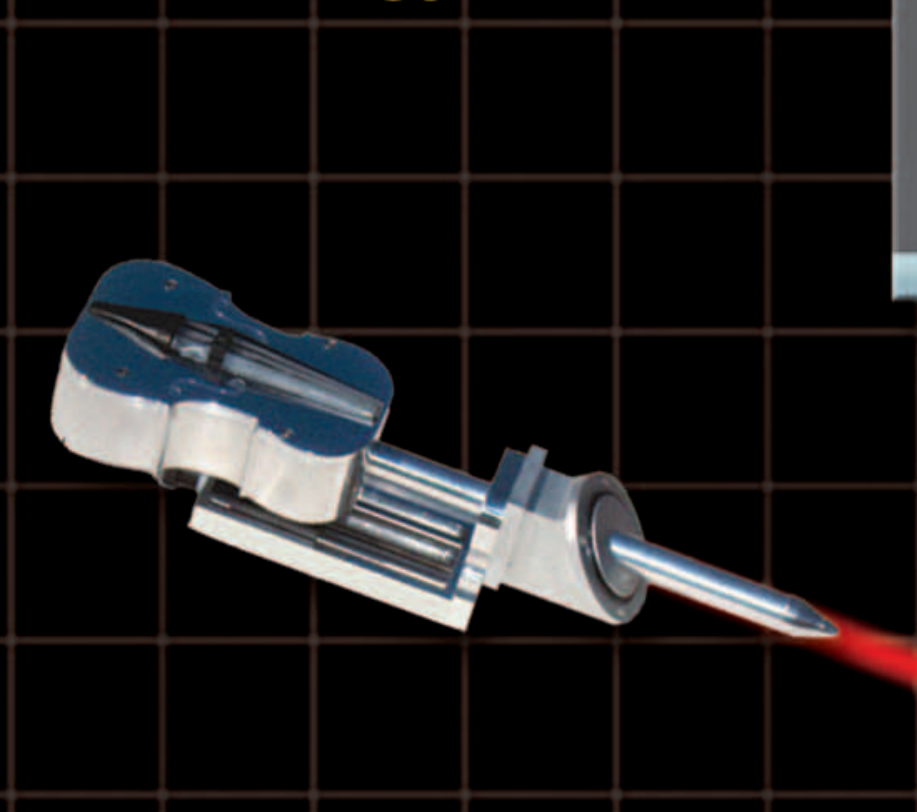

Spectral Acquisition

\section{Digital Imaging}

\section{Elemental Mapping}

\section{Indexed Mapping}

\section{NanoAnalysis for Electron Microscopy}

Evex

www.evex.com

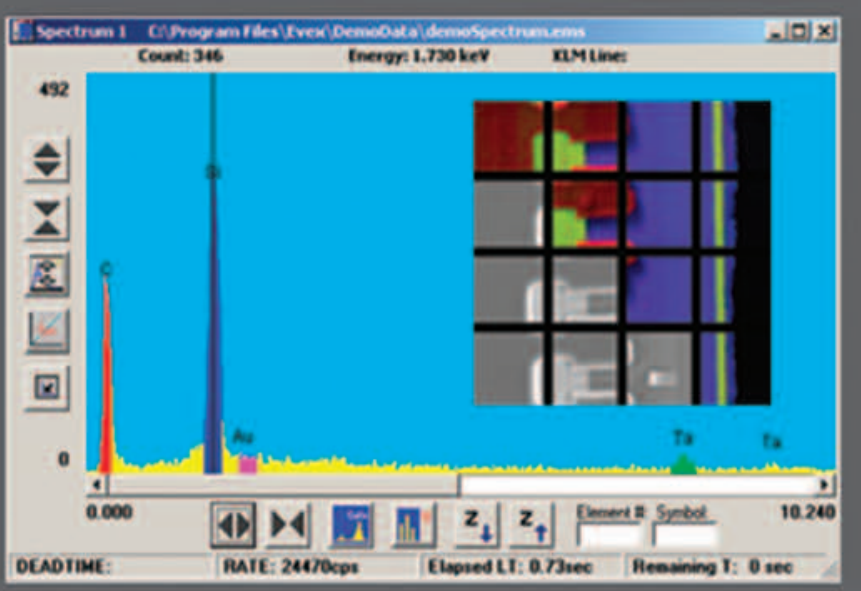

antasib
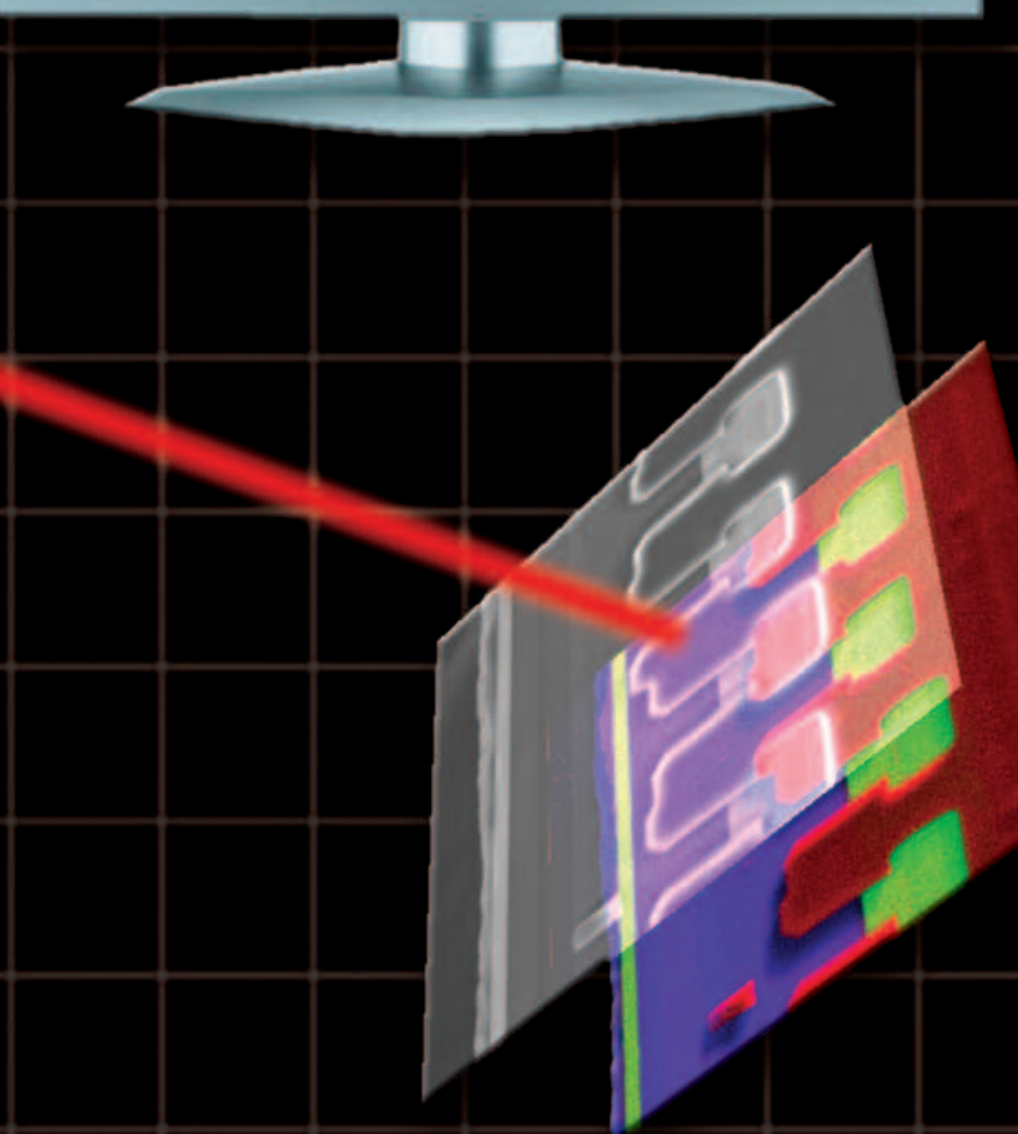

857 State Road Princeton, NJ 08540 609-252-9192 Telephone 609-252-9091 Fax 